\title{
Obtaining high degree of circular polarization at x-ray free electron lasers via a reverse undulator taper
}

\author{
E. A. Schneidmiller* and M. V. Yurkov \\ Deutsches Elektronen-Synchrotron (DESY), Notkestrasse 85, D-22607 Hamburg, Germany
}

(Received 19 August 2013; published 27 November 2013)

\begin{abstract}
Baseline design of a typical $\mathrm{x}$-ray free electron laser (FEL) undulator assumes a planar configuration which results in a linear polarization of the FEL radiation. However, many experiments at x-ray FEL user facilities would profit from using a circularly polarized radiation. As a cheap upgrade, one can consider an installation of a short helical (or cross-planar) afterburner, but then one should have an efficient method to suppress the powerful linearly polarized background from the main undulator. In this paper we propose a new method for such a suppression: an application of the reverse taper in the main undulator. We discover that in a certain range of the taper strength, the density modulation (bunching) at saturation is practically the same as in the case of a nontapered undulator while the power of linearly polarized radiation is suppressed by orders of magnitude. Then strongly modulated electron beam radiates at full power in the afterburner. Considering the SASE3 undulator of the European XFEL as a practical example, we demonstrate that soft $\mathrm{x}$-ray radiation pulses with peak power in excess of $100 \mathrm{GW}$ and an ultimately high degree of circular polarization can be produced. The proposed method is rather universal, i.e., it can be used at SASE FELs and seeded (self-seeded) FELs, with any wavelength of interest, in a wide range of electron beam parameters, and with any repetition rate. It can be used at different X-ray FEL facilities, in particular at Linac Coherent Light Source after installation of the helical afterburner in the near future.
\end{abstract}

PACS numbers: 41.60.Cr

\section{INTRODUCTION}

Successful operation of $x$-ray free electron lasers (FELs) [1-3], based on the self-amplified spontaneous emission (SASE) principle [4], opens up new horizons for photon science. One of the important requirements of FEL users in the near future will be polarization control of $\mathrm{x}$-ray radiation. Baseline design of a typical x-ray FEL undulator assumes a planar configuration which results in a linear polarization of the FEL radiation. However, many experiments at $x$-ray FEL user facilities would profit from using a circularly polarized radiation. There are different ideas [5-16] for possible upgrades of the existing (or planned) planar undulator beam lines.

As a cheap upgrade, one can consider an installation of a short helical afterburner. In particular, an electromagnetic helical afterburner will be installed behind the soft x-ray planar undulator SASE3 of the European XFEL. However, to obtain a high degree of circular polarization, one needs to suppress (or separate) powerful linearly polarized radiation from the main undulator. Different options for such a suppression (separation) are considered: using achromatic bend between planar undulator and helical afterburner $[8,9]$; tuning resonance frequency of the afterburner to

\footnotetext{
*evgeny.schneidmiller@desy.de
}

Published by the American Physical Society under the terms of the Creative Commons Attribution 3.0 License. Further distribution of this work must maintain attribution to the author(s) and the published article's title, journal citation, and DOI. the second harmonic of the planar undulator [6,10,11]; separating source positions and using slits for spatial filtering [15].

In this paper we propose a new method for suppression of the linearly polarized background from the main undulator: application of the reverse undulator taper. In particular, in the case of SASE3 undulator of the European XFEL, we demonstrate that soft $\mathrm{x}$-ray radiation pulses with peak power in excess of $100 \mathrm{GW}$ and an ultimately high degree of circular polarization can be produced. As for a comparison with the other methods, our suppression method is free, easy to implement, and the most universal: it can be used at SASE FELs and seeded (self-seeded) FELs, with any wavelength of interest, in a wide range of electron beam parameters, and with any repetition rate. It can be applied at different $x$-ray FEL facilities, in particular at Linac Coherent Light Source (LCLS) after installation of the helical afterburner in the near future.

\section{METHOD DESCRIPTION}

In a short-wavelength SASE FEL the undulator tapering is used for two purposes: to compensate an electron beam energy loss in the undulator due to the wakefields and spontaneous undulator radiation; and to increase FEL power (postsaturation taper). In both cases the undulator parameter $K$ decreases along the undulator length. The essence of our method is that we use the opposite way of tapering: parameter $K$ increases what is usually called reverse (or negative) taper. We discover that in some range 


\section{SELECTED RESULTS OF THE ONE-DIMENSIONAL THEORY}

reverse-tapered planar undulator

helical afterburner

FIG. 1. Conceptual scheme for obtaining circular polarization at $\mathrm{x}$-ray FELs.

of the taper strength, the bunching factor at saturation is practically the same as in the reference case of the nontapered undulator, the saturation length increases slightly while the saturation power is suppressed by orders of magnitude. Therefore, our scheme is conceptually very simple (see Fig. 1): in a tapered main (planar) undulator the saturation is achieved with a strong microbunching and a suppressed radiation power, then the modulated beam radiates at full power in a helical afterburner, tuned to the resonance.

Note that reverse undulator taper was considered in the past to increase saturation efficiency of FEL oscillators [17], and to produce attosecond pulses in x-ray FELs [18]. In this paper we discover a new useful feature of the reverse taper: a possibility to generate a strongly modulated electron beam at a pretty much reduced level of the radiation power.

To be specific, in this paper we will concentrate on the case of a helical afterburner and use the following formula for the degree of circular polarization:

$$
D_{\text {cir }} \simeq 1-\frac{P_{\text {lin }}}{2 P_{\text {cir }}}-F_{\mathrm{A}} \text {, }
$$

where $P_{\text {lin }}$ is the power of the linearly polarized radiation from the main undulator, $P_{\text {cir }}$ is the power of the circularly polarized radiation from the helical afterburner. A factor of 2 in the denominator is easy to understand since the linearly polarized wave can be decomposed into left and right circularly polarized waves, and we consider the case when $P_{\text {lin }} \ll P_{\text {cir }}$. Except for a contamination due to linearly polarized background from the main undulator, a decrease of $D_{\text {cir }}$ can be caused by field imperfections of the helical afterburner as well as by other sources of radiation of the modulated beam (edge radiation, coherent synchrotron radiation, etc.) having different polarization properties. We describe all these possible contributions with a separate term $F_{\mathrm{A}}$. Note that even in the case of an ideal undulator, the term $F_{\mathrm{A}}$ can be of the order of the inverse number of periods in the afterburner. Further discussions on this subject would go beyond the scope of this paper since our goal is to minimize the term $P_{\text {lin }} /\left(2 P_{\text {cir }}\right)$. We only notice here that there is not much sense to make it significantly smaller than the term $F_{\mathrm{A}}$. In most cases it means that a suppression of the term $P_{\text {lin }} /\left(2 P_{\text {cir }}\right)$ to a few per mil level is sufficient. Then we can state that a suppression scheme provides an ultimately high degree of circular polarization.
A detailed theoretical analysis of the considered effect will be published elsewhere [19]. Here we present some selected results.

Let us consider the normalized detuning parameter [20]:

$$
\hat{C}=\left(k_{\mathrm{w}}-\frac{\omega\left(1+K^{2}\right)}{2 c \gamma^{2}}\right) \Gamma^{-1} .
$$

The following notations are introduced here: $k_{\mathrm{w}}=$ $2 \pi / \lambda_{\mathrm{w}}$ is the undulator wave number, $\omega$ is the frequency of the electromagnetic wave, $K$ is the rms undulator parameter, $\gamma$ is relativistic factor, and $\Gamma$ is the gain parameter. The latter can be expressed in terms of the FEL parameter $\rho[21]: \Gamma=4 \pi \rho / \lambda_{\mathrm{w}}$.

We start our consideration with the case when a highgain FEL is coherently seeded at a given frequency $\omega$, and the undulator is not tapered. The properties of the FEL are then described by the detuning parameter (see, for example, [20] for more details). In particular, in the highgain linear regime (i.e. when the normalized undulator length $\hat{z}=\Gamma z \gg 1$ ), the squared modulus of the bunching factor $|b|^{2}$ and the normalized FEL power $\hat{\eta}=$ $P /\left(\rho P_{\text {beam }}\right)$ are of the same order when an FEL operates close to the resonance, $|\hat{C}|<1$. The normalized growth rate (inverse field gain length) of the FEL instability, $\operatorname{Re} \hat{\Lambda}=\operatorname{Re} \Lambda / \Gamma$, is of the order of unity in this regime. At the same time, the initial problem solution leads to an interesting result for a large negative detuning, $\hat{C}<0$ and $|\hat{C}| \gg 1$. In this case the bunching factor and the normalized FEL power are connected in the high-gain linear regime by a simple relation (see the Appendix):

$$
|b|^{2} \simeq|\hat{C}|^{2} \hat{\eta}
$$

i.e. the power is strongly suppressed with respect to the squared modulus of the bunching factor. Note that the tendency approximately holds at the FEL saturation. The normalized growth rate in the considered case gets smaller, $\operatorname{Re} \hat{\Lambda} \simeq|\hat{C}|^{-1 / 2}$, with the corresponding increase of the gain length (and of the saturation length). As it is discussed in the Appendix, Eq. (3) can be explained by this increase. Indeed, the proportionality between squared amplitudes of the bunching and of the field is given by the fourth power of the gain length, or the squared detuning in the considered limit.

Now let us consider a SASE FEL with linearly tapered undulator. The normalized detuning parameter changes as follows:

$$
\hat{C}(\hat{z})=\beta \hat{z}
$$

where

$$
\beta=-\frac{\lambda_{\mathrm{w}}}{4 \pi \rho^{2}} \frac{K(0)}{1+K(0)^{2}} \frac{d K}{d z},
$$

and $K(0)$ is the initial value of the rms undulator parameter. Note that as a reference frequency we always consider 
the resonance frequency at the undulator entrance. Of course, in a SASE FEL a finite frequency band is amplified, and its maximum and width can evolve along the undulator length [22].

The theory of a high-gain FEL with varying undulator parameter has been developed in [22] in the limit of a small taper strength [23], $|\beta| \ll 1$. In particular, the authors of [22] have derived corrections to the FEL growth rate up to the second order. Unfortunately, we cannot use the results of [22] for our purpose because, in the case of small corrections, the tendency we would like to demonstrate (small ratio $\hat{\eta} /|b|^{2}$ ) is not seen. For this reason we present here a result of the theory [19] that is valid in the case of a large taper strength. For a high-gain linear regime and a large negative taper strength, $\beta<0$ and $1 \ll|\beta| \ll \hat{z}$, the relation between the ensemble-averaged squared modulus of the bunching factor $\left\langle|b|^{2}\right\rangle$ and the ensemble-averaged normalized FEL power $\langle\hat{\eta}\rangle$ can be approximated as

$$
\left\langle|b|^{2}\right\rangle \simeq|\beta|^{2} \hat{z}^{2}\langle\hat{\eta}\rangle .
$$

This equation looks similar to Eq. (3) with the detuning parameter given by (4). One can see that, indeed, for large negative $\beta$ and large $\hat{z}$, the squared bunching factor is much larger than the normalized FEL power. Both quantities are proportional to $\exp (4 \sqrt{\hat{z} /|\beta|})$, i.e., they evolve along the undulator length with a decreasing growth rate.

The explanation of Eq. (6) can be done in the following way. It is known from [22] that in the case of a weak taper, the central frequency of the amplified band moves half as fast as does the resonance frequency (corresponding to the current value of the undulator parameter $K$ ). The situation is quite different in the case of strong taper [19], i.e., when $|\beta| \gg 1$. In the case of positive $\beta$ the central frequency completely follows the changes of $K$, while in the case of a reverse taper, $\beta<0$, the central frequency remains to be close to the resonance at the beginning of the undulator, i.e., it does not follow the changes of $K$ at all. In other words, in the latter case the detuning continuously increases along the undulator length. The growth rate reduces, and the radiation power is suppressed with respect to the level one would have expected at a given bunching at resonance. At the same time the bunching is strong because the gain length gets longer, see explanations of Eq. (3) and the Appendix for more detail.

The asymptote of large negative $\beta$ was considered here only for illustration of the power suppression effect. For practical applications we will restrict ourselves to moderate values of $\beta$ which allow for a significant power suppression at strong bunching and an acceptable increase of the saturation length. We are interested in the values of bunching factor and FEL power at saturation, therefore we have to use a numerical simulation code (a linear analysis is not valid at saturation). Below we will use a simplified notation $b$ instead of $\sqrt{\left\langle|b|^{2}\right\rangle}$. To make our results of 1D simulation closer to practical cases, we also introduce an energy spread with a value typical for x-ray FELs. The energy spread parameter is defined as follows [20]: $\hat{\Lambda}_{\mathrm{T}}=$ $\sigma_{\gamma} /(\gamma \rho)$ with $\sigma_{\gamma}$ being the energy spread (in units of the rest energy). In our simulations we use the value $\hat{\Lambda}_{\mathrm{T}}=0.2$. The results of simulations with a $1 \mathrm{D}$ version of the code FAST [24] are presented in Figs. 2 and 3.

In Fig. 2 we show the bunching factor and normalized FEL efficiency at saturation point which is defined here as the position where the maximum bunching is reached. One can see that for negative $\beta$ the power quickly decreases (in contrast with positive $\beta$ ) although the bunching factor changes only slightly. From Fig. 3, one can find how the saturation length depends on the taper strength. A good range of this parameter for the proposed scheme is

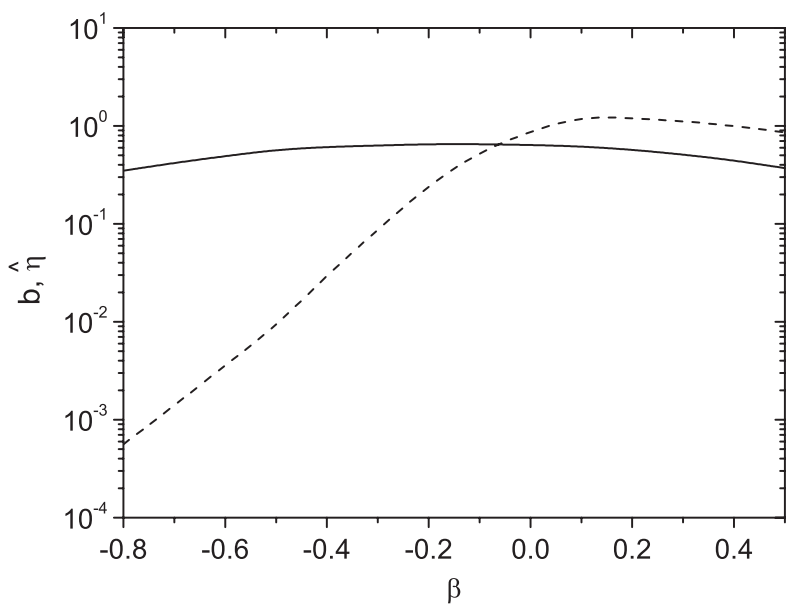

FIG. 2. Ensemble-averaged rms bunching factor (solid) and normalized FEL efficiency (dash) at saturation point (position with maximum bunching factor) versus taper strength parameter. Energy spread parameter is $\hat{\Lambda}_{\mathrm{T}}=0.2$.

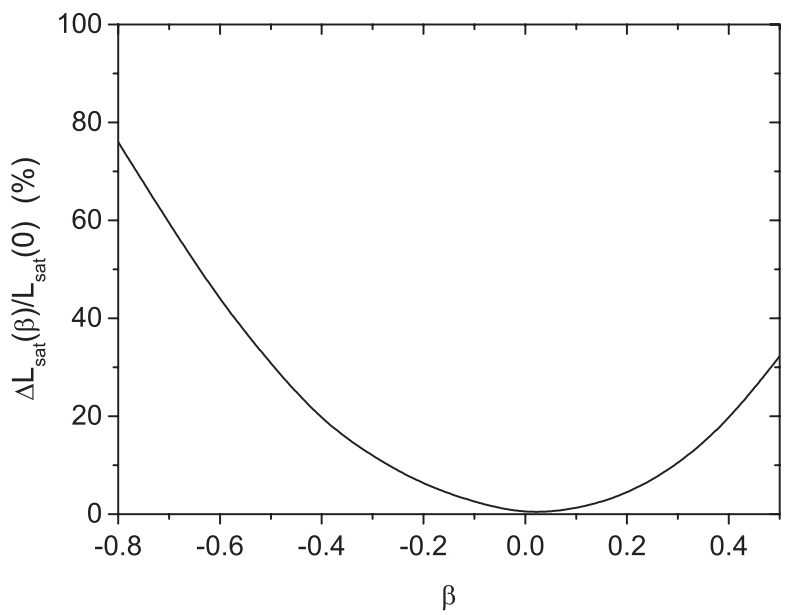

FIG. 3. Relative increase of the saturation length (defined as a length of the undulator at which maximum bunching is achieved) versus taper strength parameter. Energy spread parameter is $\hat{\Lambda}_{\mathrm{T}}=0.2$. 
$\beta \simeq-0.5-0.3$. Indeed, the bunching factor is still high in this range, there is only moderate increase of the saturation length, and the power is significantly suppressed.

\section{THREE-DIMENSIONAL SIMULATIONS FOR THE EUROPEAN XFEL}

The results of the previous section were obtained in the framework of the 1D model. We found that the reverse taper method also works well in the 3D case. We illustrate this with the parameters of the soft x-ray SASE3 undulator of the European XFEL [25]. The main parameters used in our simulations are presented in Table I. The electron beam parameters are taken from the table provided by the European XFEL beam dynamics group [26] for the bunch charge of $0.5 \mathrm{nC}$. We consider operation of SASE3 in "fresh bunch" mode [27] when the energy spread of electron bunches is not spoiled by the FEL interaction in the upstream SASE1 undulator. The simulations were performed with the 3D version of the code FAST [24].

A gap-tunable permanent-magnet SASE3 undulator consists of 21 undulator modules, each of them is $5 \mathrm{~m}$ long. One can easily control the active part of the undulator by opening the gaps of the modules which are not needed. In our case we use only 11 last modules to adapt an active undulator length to the saturation length for the given wavelength $(1.5 \mathrm{~nm})$ and electron beam parameters. A long-period electromagnetic helical afterburner is being developed [28] for installation behind the SASE3 undulator. The choice of technology is driven by the request of users to quickly change (between the macropulses, i.e.,

TABLE I. Main parameters used in simulations.

\begin{tabular}{lc}
\hline \hline Electron beam & \\
\hline Energy & $14 \mathrm{GeV}$ \\
Charge & $0.5 \mathrm{nC}$ \\
Peak current & $5 \mathrm{kA}$ \\
rms normalized slice emittance & $0.7 \mu \mathrm{m}$ \\
rms slice energy spread & $2.2 \mathrm{MeV}$ \\
Planar undulator & \\
Period & $6.8 \mathrm{~cm}$ \\
$K_{\text {rms }}$ & 5.7 \\
Beta function & $15 \mathrm{~m}$ \\
Active magnetic length & $55 \mathrm{~m}$ \\
Taper $\Delta K_{\text {rms }} / K_{\text {rms }}(0)$ & $2.1 \%$ \\
Helical afterburner & \\
Period & $16 \mathrm{~cm}$ \\
$K$ & 3.6 \\
Beta function & $15 \mathrm{~m}$ \\
Magnetic length & $10 \mathrm{~m}$ \\
Radiation & \\
Wavelength & $1.5 \mathrm{~nm}$ \\
Power from planar undulator, $P_{\text {lin }}$ & $0.4 \mathrm{GW}$ \\
Power from helical undulator, $P_{\text {cir }}$ & $155 \mathrm{GW}$ \\
$1-P_{\text {lin }} /\left(2 P_{\text {cir }}\right)$ & $99.9 \%$ \\
\hline \hline
\end{tabular}

with the frequency of $5 \mathrm{~Hz}$ ) the polarization of the output radiation between left and right.

We optimized the taper strength in the main undulator such that the radiation power is sufficiently suppressed, on the one hand, and the bunching factor is still close to that in the case of the untapered undulator, on the other hand. We ended up with a $2.1 \%$ increase of the $K$ parameter over the undulator length of $55 \mathrm{~m}$. According to (5), this corresponds to the one-dimensional [29] normalized taper strength of -0.34 .

Evolution of the bunching factor along the planar undulator and the helical afterburner is shown in Fig. 4, and the time dependence of the bunching factor at the exit of the planar undulator-in Fig. 5. One can see that the bunching factor reaches a pretty high level and becomes even larger in the helical afterburner.

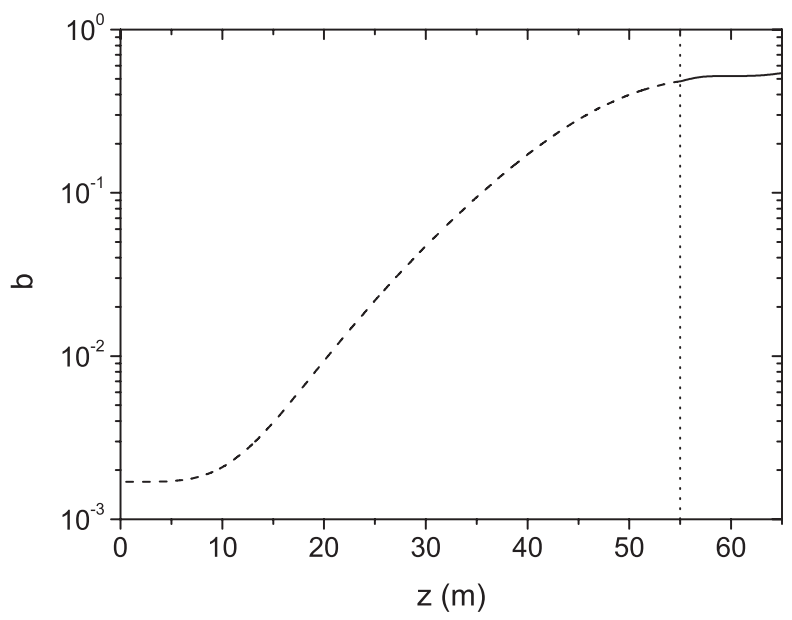

FIG. 4. Evolution of the ensemble-averaged rms bunching factor along the planar undulator SASE3 (dashed) and the helical afterburner (solid).

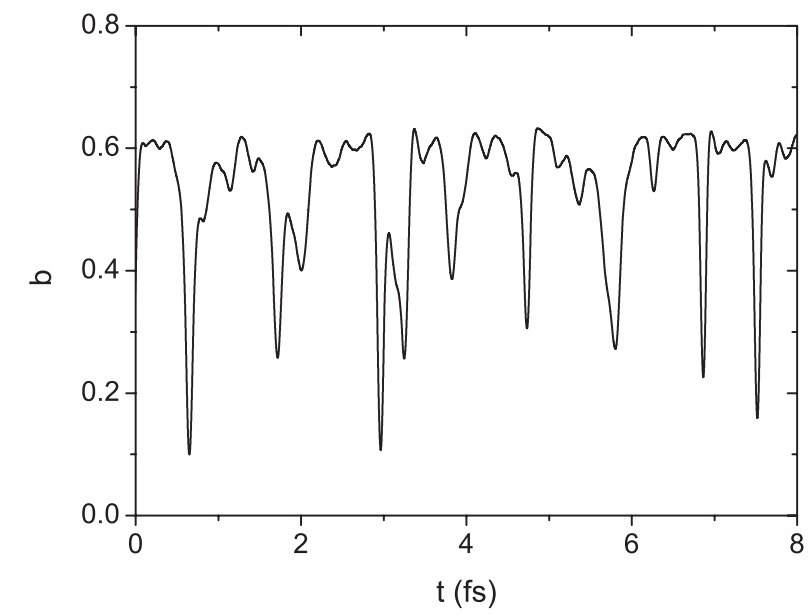

FIG. 5. Modulus of bunching factor versus time at the exit of the planar undulator SASE3 (position $55 \mathrm{~m}$ on Fig. 4). A central part of the electron bunch is shown. 


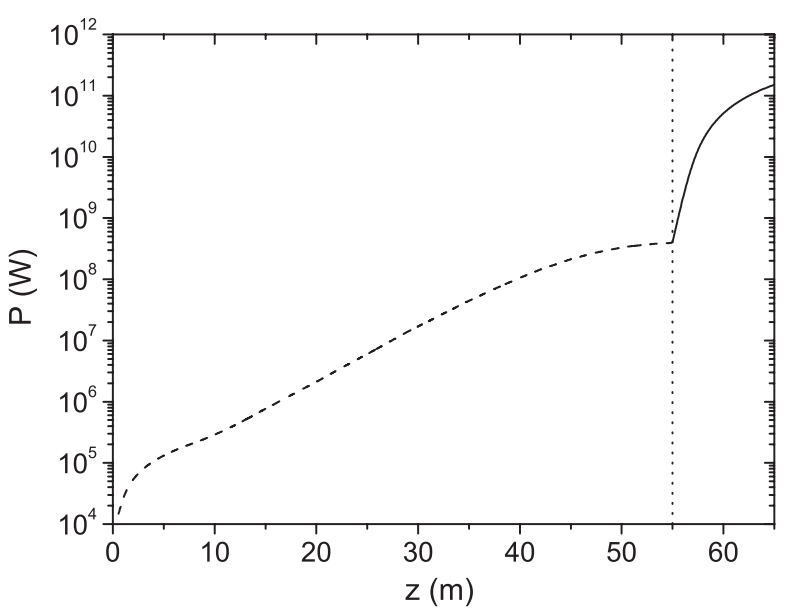

FIG. 6. FEL power versus the length of the planar main undulator SASE3 (dashed) and the helical afterburner (solid).

Radiation power as a function of position in the planar main undulator and in the helical afterburner is shown in Fig. 6. One can see that, indeed, linearly polarized radiation from the main undulator is strongly suppressed (it is about $0.4 \mathrm{GW}$ ), and the powerful circularly polarized radiation quickly builds up in the afterburner. This happens because the bunching is strongly detuned from the resonance with the last part of the planar undulator, but the $K$ value of the afterburner is optimized in such a way that it is close to the resonance, and maximum power is achieved at the end of the afterburner. A part of the radiation pulse is shown in Fig. 7 for illustration; ensemble-averaged peak power reaches $155 \mathrm{GW}$. Now we can calculate the degree of circular polarization (not considering the term $F_{\mathrm{A}}$ ) due to contamination from the planar undulator: $1-P_{\text {lin }} /\left(2 P_{\text {cir }}\right) \simeq 0.999$. Note that a further suppression of the linearly polarized background and improvement of the quantity $1-P_{\text {lin }} /\left(2 P_{\text {cir }}\right)$ is easily possible by going to a stronger taper at the price of a mild reduction of bunching

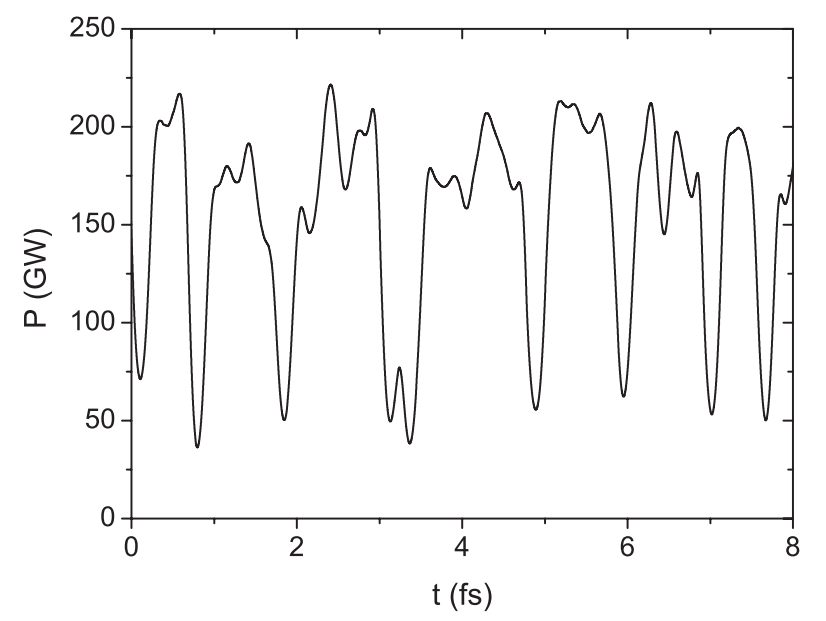

FIG. 7. Peak power of circularly polarized radiation at the exit of the afterburner (position $65 \mathrm{~m}$ on Fig. 6). A central part of the $\mathrm{X}$-ray pulse is shown. factor (and, consequently, the power of circularly polarized radiation). However, this would probably make no sense because the degree of circular polarization would be mainly defined by the term $F_{\mathrm{A}}$, see the discussion above.

Parameters of the helically polarized radiation are shown in Table I. The pulse duration and the pulse energy are defined by the chosen bunch charge (set of charges from $20 \mathrm{pC}$ to $1 \mathrm{nC}$ with different parameters will be available at the European XFEL). For example, the pulse duration can be chosen between few femtoseconds and 100 femtoseconds. In all cases the peak power and the degree of circular polarization will be comparable to those shown in Table I. Let us also notice that our method will work in a wide range of photon energies so that one can easily cover not only L-edges but also M-edges of all interesting elements. Indeed, in the considered case of lasing at $1.5 \mathrm{~nm}$ the active length of the undulator is $55 \mathrm{~m}$ (to be compared to the saturation length of $45 \mathrm{~m}$ for the untapered case, i.e., we have only $20 \%$ increase in length). The total magnetic length of the SASE3 undulator is $105 \mathrm{~m}$ so that there is a big reserve for going to shorter wavelength. Generally speaking, our method can also work at hard x-ray beam lines if this is requested by users.

We should also comment on the expected stability of the degree of circular polarization. Since SASE pulse energy fluctuates shot to shot, the degree of circular polarization is not going to be perfectly stable. However, the relative contribution of "wrong polarization" $P_{\text {lin }} /\left(2 P_{\text {cir }}\right)$ is on the order of $10^{-3}$, and the SASE fluctuations at saturation are typically below $10 \%$. Thus, fluctuations of the timeintegrated ratio $P_{\text {lin }} /\left(2 P_{\text {cir }}\right)$ would be about $10^{-4}$ assuming that the $P_{\text {lin }}$ and $P_{\text {cir }}$ are uncorrelated. The latter assumption is, however, not true since both polarizations are produced by the same electron bunch with the same realization of microbunching distribution along the bunch (even though it slightly evolves at saturation). Thus, we expect strong correlations, and the fluctuations of the considered ratio will be further suppressed. Our crude estimate is that the degree of circular polarization, determined by our suppression scheme, would fluctuate shot to shot at the level of $10^{-5}$ for the parameter set considered in this section.

Finally, let us note that in the case of energy loss along the undulator due to the wakefields and spontaneous undulator radiation at high energies, the strength of the reverse taper can be decreased in accordance with formula (7), see discussion below. In our case both effects are small corrections, each of them is on the order of $0.1 \%$ in the active part of the SASE3 undulator-to be compared with about $2 \%$ of the $K$ change.

\section{DISCUSSION}

For simplicity we have considered up to now the case when only the undulator parameter $K$ changes linearly along the undulator length. Obviously, the parameter $\beta$ can be generalized to the case when, in addition, the mean 
energy of electrons changes due to the wakefields and spontaneous undulator radiation:

$$
\beta=-\frac{\lambda_{\mathrm{w}}}{4 \pi \rho^{2}}\left[\frac{K(0)}{1+K(0)^{2}} \frac{d K}{d z}-\frac{1}{\gamma(0)} \frac{d \gamma}{d z}\right]
$$

Here $\gamma(0)$ is the gamma factor at the undulator entrance. If the energy loss is not negligible, one should decrease the taper strength correspondingly.

It was shown in [18] that the action of an energy chirp on FEL gain is equivalent to the action of the corresponding undulator tapering. Here we notice that the effect, described in this paper (drastic suppression of FEL power at a relatively strong microbunching via a reverse taper), also takes place in the case of a negative chirp parameter. If the influence of the energy chirp cannot be neglected, the formula (7) can be easily generalized for inclusion of the latter effect, see [18] for more details. We would also like to note here that the use of an energy chirp for suppression of radiation power while keeping a high level of microbunching can find interesting applications.

We have mainly considered the case of a SASE FEL in this paper. In the case of seeded (self-seeded) FELs, one can use two modifications of the suppression method: with reverse taper or with constant detuning of the $K$ parameter (so that the detuning parameter $\hat{C}$ is negative).

We have simulated the helical afterburner for the SASE3 undulator of the European XFEL. Obviously, as an afterburner one can also consider a cross-planar undulator with a phase shifter $[5,11]$ which may give more possibilities for polarization control (see Fig. 8). In this case the length of the afterburner should be short enough so that density modulation stays almost unchanged as the beam propagates in the afterburner (and this is easier in the scheme with reverse taper since the energy modulation is weak despite the beam is fully bunched). A more complicated cascaded crossed undulator $[13,14]$ can be used as well.

Finally, we note that the suppression scheme, proposed in this paper, can be tested and used soon at the Linac Coherent Light Source (LCLS) [2]. A fixed-gap planar undulator is used at LCLS to generate hard and soft$\mathrm{x}$-ray radiation. A helical afterburner is going to be installed in order to provide a circular polarization for user operation at LCLS [30]. Design of the planar undulator allows for a mild tapering by making use of canted poles. This option is normally used for compensation of the beam energy loss along the undulator length, and for the postsaturation taper-in both cases a standard (positive)

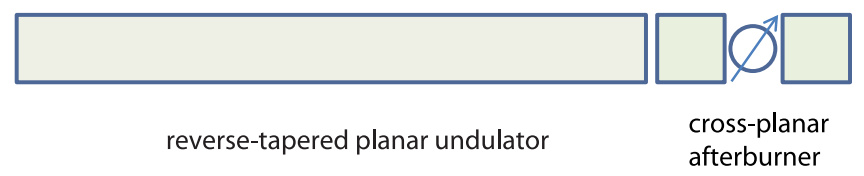

FIG. 8. Scheme for obtaining circular polarization in a crossplanar undulator. sign of taper is needed. We propose here to use a reverse taper to obtain powerful x-ray radiation (in soft and hard $\mathrm{x}$-ray regimes) with a high degree of circular polarization, in excess of $99 \%$. Our estimates with the help of the formula (5) suggest that the strength of the reverse taper should typically be on the order of $1 \%$ over active undulator length. After optimizing the taper strength and active length of the main undulator, the $K$ value of the helical afterburner should be scanned in order to obtain maximum power. Such an experiment can be performed in the near future.

\section{ACKNOWLEDGMENTS}

We would like to thank R. Brinkmann, V. Balandin, N. Golubeva, W. Decking, and T. Limberg for useful discussions.

\section{APPENDIX: FEL POWER AND BUNCHING AT A LARGE NEGATIVE DETUNING}

We consider here the relation between FEL power and bunching in the 1D, cold beam case. The theoretical description of a high-gain FEL and the notations are close to those presented in [20]. The evolution of the electric field of the amplified electromagnetic wave is governed by the following equation:

$$
\tilde{E}^{\prime \prime \prime}+2 \mathrm{i} \hat{C} \tilde{E}^{\prime \prime}-\hat{C}^{2} \tilde{E}^{\prime}=\mathrm{i} \tilde{E} .
$$

This equation is valid for the general case of initial conditions for the electron beam and the radiation at the undulator entrance. The normalized detuning parameter is given by Eq. (2), and the prime denotes differentiation with respect to $\hat{z}$. Equation (A1) is a linear ordinary differential equation with constant coefficients, so its general solution is given by a superposition of three linearly independent solutions:

$$
\tilde{E}=\sum_{j=1}^{3} C_{j} \exp \left(\lambda_{j} \hat{z}\right),
$$

where the $C_{j}$ are constants. The eigenvalues $\lambda_{j}$ are the solutions of the eigenvalue equation

$$
\lambda(\lambda+\mathrm{i} \hat{C})^{2}=\mathrm{i}
$$

The field amplitude and its derivatives at any longitudinal $\hat{z}$ coordinate are calculated as follows:

$$
\left[\begin{array}{c}
\tilde{E} \\
\tilde{E}^{\prime} \\
\tilde{E}^{\prime \prime}
\end{array}\right]_{\hat{z}}=M(\hat{z} \mid 0)\left[\begin{array}{c}
\tilde{E} \\
\tilde{E}^{\prime} \\
\tilde{E}^{\prime \prime}
\end{array}\right]_{0},
$$

where

$$
\tilde{E}(0), \quad \tilde{E}^{\prime}(0), \quad \tilde{E}^{\prime \prime}(0),
$$

are the field amplitude and its first and second derivatives with respect to $\hat{z}$ at the undulator entrance at $\hat{z}=0$, and the transfer matrix $M(\hat{z} \mid 0)$ is 


$$
M=\left[\begin{array}{ccc}
\tilde{E}_{1} & \tilde{E}_{2} & \tilde{E}_{3} \\
\tilde{E}_{1}^{\prime} & \tilde{E}_{2}^{\prime} & \tilde{E}_{3}^{\prime} \\
\tilde{E}_{1}^{\prime \prime} & \tilde{E}_{2}^{\prime \prime} & \tilde{E}_{3}^{\prime \prime}
\end{array}\right]_{\hat{z}} \times\left[\begin{array}{ccc}
\tilde{E}_{1} & \tilde{E}_{2} & \tilde{E}_{3} \\
\tilde{E}_{1}^{\prime} & \tilde{E}_{2}^{\prime} & \tilde{E}_{3}^{\prime} \\
\tilde{E}_{1}^{\prime \prime} & \tilde{E}_{2}^{\prime \prime} & \tilde{E}_{3}^{\prime \prime}
\end{array}\right]_{0}^{-1}
$$

The explicit expressions for the matrix elements $M_{i j}$ are

$$
\begin{aligned}
& M_{11}=\lambda_{2} \lambda_{3} B_{1}+\lambda_{1} \lambda_{3} B_{2}+\lambda_{1} \lambda_{2} B_{3}, \\
& M_{12}=-\left(\lambda_{2}+\lambda_{3}\right) B_{1}-\left(\lambda_{1}+\lambda_{3}\right) B_{2}-\left(\lambda_{1}+\lambda_{2}\right) B_{3}, \\
& M_{13}=B_{1}+B_{2}+B_{3}, \\
& M_{21}=\lambda_{1} \lambda_{2} \lambda_{3} M_{13}, \\
& M_{22}=-\lambda_{1}\left(\lambda_{2}+\lambda_{3}\right) B_{1}-\lambda_{2}\left(\lambda_{1}+\lambda_{3}\right) B_{2}-\lambda_{3}\left(\lambda_{1}+\lambda_{2}\right) B_{3}, \\
& M_{23}=\lambda_{1} B_{1}+\lambda_{2} B_{2}+\lambda_{3} B_{3}, \\
& M_{31}=\lambda_{1} \lambda_{2} \lambda_{3} M_{23}, \\
& M_{32}=-\lambda_{1}^{2}\left(\lambda_{2}+\lambda_{3}\right) B_{1}-\lambda_{2}^{2}\left(\lambda_{1}+\lambda_{3}\right) B_{2}-\lambda_{3}^{2}\left(\lambda_{1}+\lambda_{2}\right) B_{3}, \\
& M_{33}=\lambda_{1}^{2} B_{1}+\lambda_{2}^{2} B_{2}+\lambda_{3}^{2} B_{3},
\end{aligned}
$$

where

$$
\begin{aligned}
& B_{1}=\frac{\exp \left(\lambda_{1} \hat{z}\right)}{\left(\lambda_{1}-\lambda_{2}\right)\left(\lambda_{1}-\lambda_{3}\right)}, \\
& B_{2}=\frac{\exp \left(\lambda_{2} \hat{z}\right)}{\left(\lambda_{2}-\lambda_{1}\right)\left(\lambda_{2}-\lambda_{3}\right)}, \\
& B_{3}=\frac{\exp \left(\lambda_{3} \hat{z}\right)}{\left(\lambda_{3}-\lambda_{1}\right)\left(\lambda_{3}-\lambda_{2}\right)} .
\end{aligned}
$$

The derivatives $\tilde{E}^{\prime}(\hat{z})$ and $\tilde{E}^{\prime \prime}(\hat{z})$ can be expressed in terms of bunching $b(\hat{z})$ and the complex amplitude of the harmonic of energy modulation (see [20]) that is denoted here as $b_{P}(\hat{z})$ :

$$
\tilde{E}^{\prime} / E_{0}=-2 b, \quad \tilde{E}^{\prime \prime} / E_{0}=2 \mathrm{i}\left(\hat{C} b-b_{P}\right),
$$

where $E_{0}$ is the normalizing factor for the field amplitude [20].

We are interested in the relation between $\tilde{E}^{\prime}(\hat{z}), b(\hat{z})$, and $b_{P}(\hat{z})$. In the high-gain linear regime the relation does not depend on initial conditions. Nevertheless, for the sake of compactness, we consider a specific kind of the initial conditions when only a monochromatic density modulation exists at the undulator entrance. The initial conditions at $\hat{z}=0$ are then written as

$$
\tilde{E}(0)=0, \quad \tilde{E}^{\prime}(0) / E_{0}=-2 b(0), \quad \tilde{E}^{\prime \prime}(0) / E_{0}=2 i \hat{C} b(0),
$$

and the radiation field and its derivatives at any position $\hat{z}$ are given by the expressions

$$
\begin{gathered}
\tilde{E}(\hat{z}) / E_{0}=-2 M_{12}(\hat{z} \mid 0) b(0)+2 \mathrm{i} \hat{C} M_{13}(\hat{z} \mid 0) b(0), \\
\tilde{E}^{\prime}(\hat{z}) / E_{0}=-2 M_{22}(\hat{z} \mid 0) b(0)+2 \mathrm{i} \hat{C} M_{23}(\hat{z} \mid 0) b(0), \\
\tilde{E}^{\prime \prime}(\hat{z}) / E_{0}=-2 M_{32}(\hat{z} \mid 0) b(0)+2 \mathrm{i} \hat{C} M_{33}(\hat{z} \mid 0) b(0) .
\end{gathered}
$$

It is well known that a solution of the eigenvalue equation (A2) with $\operatorname{Re} \lambda>0$ exists if $\hat{C}<1.89$. We denote this solution with $\lambda_{1}$. In the high-gain linear regime, $\lambda_{1} \hat{z} \gg 1$, the term $B_{1}$ is much larger than the terms $B_{2}$ and $B_{3}$. The equations (A8) can then be simplified as follows:

$$
\begin{aligned}
\tilde{E}(\hat{z}) / E_{0} & =2 B_{1}\left(\lambda_{2}+\lambda_{3}+2 \mathrm{i} \hat{C}\right) b(0), \\
\tilde{E}^{\prime}(\hat{z}) / E_{0} & =\lambda_{1} \tilde{E}(\hat{z}) / E_{0}, \\
\tilde{E}^{\prime \prime}(\hat{z}) / E_{0} & =\lambda_{1}^{2} \tilde{E}(\hat{z}) / E_{0} .
\end{aligned}
$$

Using (A6), we can obtain

$$
\begin{aligned}
\tilde{E}(\hat{z}) / E_{0} & =2 B_{1}\left(\lambda_{2}+\lambda_{3}+2 \mathrm{i} \hat{C}\right) b(0), \\
b(\hat{z}) & =-\frac{1}{2} \lambda_{1} \tilde{E}(\hat{z}) / E_{0}, \\
b_{P}(\hat{z}) & =\frac{\mathrm{i}}{2} \lambda_{1}\left(\lambda_{1}+\mathrm{i} \hat{C}\right) \tilde{E}(\hat{z}) / E_{0} .
\end{aligned}
$$

Now let us consider the case of a large negative detuning: $\hat{C}<0$ and $|\hat{C}| \gg 1$. The asymptotic solution for the growing root of Eq. (A2) is

$$
\lambda_{1} \simeq \frac{1}{\sqrt{|\hat{C}|}}+\mathrm{i}|\hat{C}|,
$$

and the relations between the field, the density bunching, and energy modulation amplitude become particularly simple (we keep here only leading terms in $|\hat{C}|$ ):

$$
b(\hat{z}) \simeq-\mathrm{i}|\hat{C}| \frac{\tilde{E}(\hat{z})}{2 E_{0}}, \quad b_{P}(\hat{z}) \simeq-\sqrt{|\hat{C}|} \frac{\tilde{E}(\hat{z})}{2 E_{0}} .
$$

We can also present here the relations between squared amplitudes. The normalized FEL power (see Sec. III) is connected with the field amplitude as [20]

$$
\hat{\eta}=\frac{1}{4} \frac{|\tilde{E}|^{2}}{E_{0}^{2}}
$$

Then we obtain compact relations:

$$
|b|^{2} \simeq|\hat{C}|^{2} \hat{\eta}, \quad\left|b_{P}\right|^{2} \simeq|\hat{C}| \hat{\eta} .
$$

When a high-gain FEL operates close to the resonance $(\hat{C}<1)$, the normalized field, bunching, and amplitude of energy modulation are of the same order. In the case of a large negative detuning, however, the field is suppressed. This should not be considered as a paradox. Indeed, according to a mechanism of a high-gain FEL, the field modulates the beam in energy on the scale of the gain length. In other words, the amplitude of energy modulation in the exponential gain regime must be proportional to the product of the gain length and the field. The energy modulations are converted into the density bunching through the $R_{56}$ of the undulator also on the scale of the gain length (i.e. the integration over $\hat{z}$ is done once more), so that the density bunching is proportional to the product of the field and the squared gain length. The normalized field gain 
length $L_{g} \Gamma=\left(\operatorname{Re} \lambda_{1}\right)^{-1}$ is on the order of unity when FEL operates close to the resonance, but in the considered limit it equals $\sqrt{|\hat{C}|}$. So, Eqs. (A12) are in agreement with the qualitative considerations given above. At the same time, due to a strong detuning from resonance, the modulated beam radiates a weaker field. Thus, the selfconsistency of the FEL model is preserved in the considered limit, and there is no paradox. If one then considers the proportionality between squared amplitudes of the bunching and of the field, it is given by the fourth power of gain length, or the squared detuning in the considered limit.

[1] W. Ackermann et al., Nat. Photonics 1, 336 (2007).

[2] P. Emma et al., Nat. Photonics 4, 641 (2010).

[3] T. Ishikawa et al., Nat. Photonics 6, 540 (2012).

[4] A. M. Kondratenko and E. L. Saldin, Part. Accel. 10, 207 (1980).

[5] K.-J. Kim, Nucl. Instrum. Methods Phys. Res., Sect. A 445, 329 (2000).

[6] Z. Huang and S. Reiche, in Proceedings of FEL2004 Conference (Comitato Conferenze Elettra, Trieste, Italy, 2004), p. 201, [http://www.jacow.org].

[7] Y. Ding and Z. Huang, Phys. Rev. ST Accel. Beams 11, 030702 (2008).

[8] Y. Li et al., in Proceedings of the 11th European Particle Accelerator Conference, Genoa, 2008 (EPS-AG, Genoa, Italy, 2008), WEPC118 [http://www.jacow.org].

[9] Y. Li, W. Decking, B. Faatz, and J. Pflueger, Phys. Rev. ST Accel. Beams 13, 080705 (2010).

[10] H. Geng, Y. Ding, and Z. Huang, Nucl. Instrum. Methods Phys. Res., Sect. A 622, 276 (2010).

[11] E. A. Schneidmiller and M. V. Yurkov, in Proceedings of the 32nd Free Electron Laser Conference, Malmö, Sweden (Max-lab, Sweden, 2010), p. 123 [http://www.jacow.org].
[12] E. A. Schneidmiller and M. V. Yurkov, in Proceedings of the 32nd Free Electron Laser Conference, Malmö, Sweden (Ref. [11]), p. 115, [http://www.jacow.org].

[13] T. Tanaka and H. Kitamura, AIP Conf. Proc. 705, 231 (2004).

[14] G. Geloni, V. Kocharyan, and E. Saldin, Report No. DESY-11-009, 2011.

[15] G. Geloni, V. Kocharyan, and E. Saldin, Report No. DESY-11-096, 2011.

[16] Zhang Tong et al., arXiv:1301.3597.

[17] E. L. Saldin, E. A. Schneidmiller, and M. V. Yurkov, Opt. Commun. 103, 297 (1993).

[18] E. L. Saldin, E. A. Schneidmiller, and M. V. Yurkov, Phys. Rev. ST Accel. Beams 9, 050702 (2006).

[19] E. A. Schneidmiller and M. V. Yurkov (unpublished).

[20] E. L. Saldin, E. A. Schneidmiller, and M. V. Yurkov, The Physics of Free Electron Lasers (Springer, Berlin, 1999).

[21] R. Bonifacio, C. Pellegrini, and L. M. Narducci, Opt. Commun. 50, 373 (1984).

[22] Z. Huang and G. Stupakov, Phys. Rev. ST Accel. Beams 8, 040702 (2005).

[23] More strictly, the condition $|\beta| \hat{z} \ll 1$ is necessary.

[24] E. L. Saldin, E. A. Schneidmiller, and M. V. Yurkov, Nucl. Instrum. Methods Phys. Res., Sect. A 429, 233 (1999).

[25] M. Altarelli et al., DESY Technical Design Report No. DESY 2006-097, 2006; see also http://xfel.desy.de.

[26] W. Decking, M. Dohlus, T. Limberg, and I. Zagorodnov (private communication).

[27] R. Brinkmann, E. A. Schneidmiller, and M. V. Yurkov, Nucl. Instrum. Methods Phys. Res., Sect. A 616, 81 (2010).

[28] N. Vinokurov et al. (private communication).

[29] Note that in the considered case the one-dimensional normalization is not very convenient since the diffraction effects play a major role. If 1D parameter $\rho$ in (5) is substituted by the corresponding 3D parameter [20], the taper strength is then -0.4 .

[30] E. Allaria et al., in Proceedings of the FEL2011 Conference, Shanghai, China, 2011, p. 31 [http://www .jacow.org]. 